\title{
Development of zoledronic acid functionalized hydroxyapatite loaded polymeric nanoparticles for the treatment of osteoporosis
}

\author{
YUNLU CAI ${ }^{*}$, TIANTIAN GAO* ${ }^{*}$ SHIPING FU and PING SUN \\ Department of Orthopedics, The Eighth People's Hospital of Shanghai, Shanghai 200235, P.R. China
}

Received March 31, 2017; Accepted September 29, 2017

DOI: $10.3892 /$ etm.2018.6263

\begin{abstract}
The present study formulated a hydroxyapatite (HA)-coated lipid nanoparticle loaded with zoledronic acid to treat patients with osteoporosis (OP). HA-coated zoledronic acid loaded lipid bilayer nanoparticles (HZL NPs) were prepared using methods of extrusion and precipitation. Nanosized particles were prepared with the aim of increasing gradual and prolonged drug release and inducing toxicity of osteoblasts. Cellular morphology was investigated by scanning electron microscopy, which revealed clear spherical shaped NPs $\sim 200 \mathrm{~nm}$ in size that could treat osteopores in the bone. In addition, a typical biphasic release pattern was observed that could be attributed to the presence of drug on the outer surface as well as on the inner core of the NPs. There was sustained release behavior $[38.17 \pm 2.12 \%(\mathrm{pH} \mathrm{7.4)}$ and $64.2 \pm 3.75 \%$ $(\mathrm{pH} 5)$ at $48 \mathrm{~h}$ ] of the drug that maintained the drug reservoir effect at the bone site. Furthermore, HZL NPs increased the cytotoxicity of HFOb 1.19 cells and increased the proportion of cells in the early $(18.1 \pm 12.4 \%)$, late $(28.7 \pm 3.7 \%)$ and necrotic $(67.5 \pm 1.2 \%)$ phases of apoptosis. Most importantly, HZL in the lipid nanoparticle exhibited a strong affinity towards HA, further enhancing its efficacy in the treatment of OP.
\end{abstract}

\section{Introduction}

Osteoporosis (OP) is a bone disease characterized by a low bone mass and deterioration of bone tissue, leading to increased bone fragility and increasing the susceptibility of patients to fracture (1). OP commonly affects the elderly and its prevalence and associated complications are rapidly increasing worldwide (1). It is associated with a reduction in bone density, which increases bone fragility and the probability of fracture, particularly of the

Correspondence to: Dr Ping Sun, Department of Orthopedics, The Eighth People's Hospital of Shanghai, 8 Cao Bao Road, Shanghai 200235, P.R. China

E-mail: pingsun347@gmail.com

*Contributed equally

Key words: zoledronic acid, osteoporosis, hydroxyapatite, nanoparticles, osteoblasts hip, wrist and spine. It is estimated that $>200$ million people have $\mathrm{OP}$ and it has been predicted that the number of those experiencing hip fractures may rise to 6.26 million by $2050(2,3)$. Current therapeutic strategies to treat the bone degradation that occurs as a result of OP include the use of bisphosphonates that increase bone mass, strength and turnover.

Zoledronic acid (ZA) is a potent antiresorptive bisphosphonate that actively inhibits osteoclast proliferation, reduces the risk of fractures in patients with postmenopausal conditions and is therefore used to treat OP (4). Bisphosphonates with nitrogen containing side chains (N-BPs) inhibit a key enzyme in the mevalonate pathway, farnesyl pyrophosphate synthase, thereby preventing the prenylation of small GTPases in osteoclasts (5). The enhanced permeation and retention effect of the nanoparticle drug delivery system also increases the accumulation of ZA in osteoclasts and inhibits the disruption of mature osteoclast activity that causes cellular apoptosis $(5,6)$. Accumulation of ZA in the bone may pose a serious health risk. It has been suggested that nanotechnologies and nanomaterials may be used to formulate alternative treatments of OP and its complications (7). In addition, it has been demonstrated that bisphosphonates inhibit bone resorption at sites of high osteoclast activity and they have therefore been extensively used to treat patients with OP (8).

Nanoparticles are making a notable impact on the medical landscape. Numerous examples of therapeutic strategies involving nanoparticles, including biocompatible drug carriers, are currently undergoing clinical trials and may be developed as more beneficial alternatives to traditional therapies (9-11). Liposomes are artificially constructed nanostructures and have been extensively studied due to their superior properties over other nanosystems. These include minimal toxicity, no immunogenicity, good biodegradability and biocompatibility, making them ideal drug delivery carriers (12-15). In addition, phospholipid bilayers allow liposomes to encapsulate hydrophobic and hydrophilic bioactive molecules. Nanocarriers with bone-targeting ligands may therefore be developed as a promising solution to deliver drugs to affected sites (16-18).

Hydroxyapatite (HA) is widely used as a naturally occurring inorganic bone substitute material due to its properties of biocompatibility and osteoconductivity. This is due to its similarity with the mineral components of bone and it is therefore able to induce local deposition/accumulation in the bone tissue $(19,20)$. It has been demonstrated that, unlike bisphosphonates, HA does not stimulate negative osteoclast 
activity; however, it stimulates positive bone remodeling and osteogenic activity (21). Following administration of such drug-loaded nanocarriers that form strong interactions with $\mathrm{HA}$, the rapid retention and accumulation of nanocarriers onto the bone tissue may occur. However, the construction of synthetic bone graft substitutes to complement the ability of the bones to self-repair is challenging $(22,23)$.

In the present study, HA-coated ZA-loaded lipid bilayer nanoparticles (HZL NPs) were designed and constructed. ZA was encapsulated in the hydrophilic cores of nanoparticles and, as HA is negatively charged, it was able to electrostatically interact with the surface of the positively charged lipid molecules. Therefore, HZL had a dual function: ZA was present in the inner core of the liposome to facilitate sustained drug release and HA was present in the outer layer to provide osteoconductivity for bone cells. Therefore, several physicochemical parameters were characterized in the present study and the affinity of nanoparticles towards HA was evaluated using the human osteoblast cell line HFOb 1.19. The results of the present study indicate that this bone-targeting drug delivery system may be developed as a novel therapeutic strategy to treat patients with OP.

\section{Materials and methods}

Reagents. ZA was purchased from Sigma-Aldrich; Merck KGaA (Darmstadt, Germany). L- $\alpha$-phosphatidylcholine (EPC), 1,2-dioleoyl-3-trimethylammonium-propane (DOTAP), and cholesterol (CHOL) were purchased from Avanti Polar Lipids Inc. (Alabaster, AL, USA). HA powder with small particle sizes was obtained from Oerlitken Metco Switzerland (Pfaffikon, Switzerland). The human osteoblast cell line HFOb 1.19 was purchased from the American Type Culture Collection (Manassas, VA, USA). All chemicals were of analytical grade and used without further purification.

Preparation of HZL NPs. Liposomes consisting of EPC/DOTAP/CHOL (3:1:1) were prepared by hydration of a thin lipid film followed by extrusion. The lipid mixture was dissolved in $1 \mathrm{ml}$ chloroform/methanol (2:1 v/v) mixture, the resulting solution was added to a round-bottom flask and the solvent was removed using a rotary evaporator. Subsequently, the lipid film was hydrated with $3 \mathrm{mg}$ ZA and $1 \mathrm{ml} 0.2 \mu \mathrm{m}$ filtered distilled water, and the resulting suspension was gently mixed in the presence of glass beads until the lipid layer was removed from the glass wall. Following this step, the flask was left at room temperature for a further $2 \mathrm{~h}$. The liposome suspension was then extruded using an extruder system (Avanti Wind Systems, Franklin, WI, USA), which repeatedly passed the suspension under a nitrogen atmosphere through polycarbonate membranes with decreasing pore sizes.

Briefly, 0.020 M calcium acetate and $0.010 \mathrm{M}$ phosphoric acid solutions were added to the liposomal solution (1:1:1 v/v \%), followed by the addition of an ammonium solution (20\% v/v) to adjust the $\mathrm{pH}$ to 9.0 (19). The mixture was then incubated to form a HA coating on the liposomal surface which finally resulted in HA-coated ZA-loaded lipid layer nanoparticles (HZLs) were then isolated using the Sephadex G-50 column (GE Healthcare, Chicago, IL, USA). Following preparation, liposomes were stored at $4^{\circ} \mathrm{C}$. Each formulation was prepared in triplicate.
Particle size and measurement of $\xi$-potential. The mean diameter and polydispersity of nanoparticles was measured using a dynamic light scattering technique. A Zetasizer Nano ZS (Malvern Instruments, Ltd., Malvern, UK) was used to measure the size at room temperature, with a detection angle of $90^{\circ}$. $\zeta$-potential measurements of the formulation were determined using a detection angle of $17^{\circ}$. Each sample was diluted 10 -fold with filtered water prior to each measurement. All values were calculated as the mean of three separate batches.

Morphological characterization. The morphology of the samples was characterized using a JSM field emission scanning electron microscope (JEOL Ltd., Tokyo, Japan). Powdered nanoparticles were spread onto a carbon tape over a stub and vacuum-dried. Gold coating was applied using an ion-sputtering device.

Encapsulation and loading efficiency. Quantitative analysis of ZA was performed by high-performance liquid chromatography (HPLC) (Agilent Technologies, Inc., Santa Clara, CA, USA) using a C18 (1) column ( $3 \mu \mathrm{m}, 150 \times 4.6 \mathrm{~mm})$ (Agilent). The injection volume used was $20 \mu \mathrm{l}$ with a $1 \mathrm{ml} / \mathrm{min}$ flow rate using a mobile phase 20:80 (v/v) of acetonitrile and an aqueous solution ( $8 \mathrm{mM}$ di-potassium hydrogen orthophosphate, $2 \mathrm{mM}$ di-sodium hydrogen orthophosphate and $7 \mathrm{mM}$ tetra-n-butyl ammonium hydrogen sulfate, adjusted at $\mathrm{pH} 7.0$ with sodium hydroxide). Briefly, the drug-loaded nanocarrier was filtered with an Amicon centrifugal filter (Sigma-Aldrich; Merck $\mathrm{KGaA}$ ) by centrifugation at a high speed of 2,571 x g for $20 \mathrm{~min}$ at $37^{\circ} \mathrm{C}$. The unencapsulated ZA was analyzed at $215 \mathrm{~nm}$ to quatify the amount to be loaded in the nanoparticle using equations 1 and 2. Furthermore, a standard curve of ZA was individually plotted. The ZA encapsulation efficiency was calculated using the following equations:

Equation 1

Encapsulation efficiency $=\frac{\text { Total amount of ZA }- \text { Amount of free ZA } \times 100}{\text { Total amount of ZA }}$

Equation 2

$$
\text { Loading efficiency }=\frac{\text { Total amount of ZA }- \text { Amount of free ZA x100 }}{\text { Total weight of nanoparticles }}
$$

In vitro release studies. To investigate the release of $\mathrm{ZA}$ from HZL NPs, a fixed amount $(1 \mathrm{mg} / \mathrm{ml})$ of drug-loaded nanoparticles was incubated in $10 \mathrm{ml}$ phosphate buffer solution [PBS (pH 7.4) ionic strength $0.1 \mathrm{M}$ with $0.1 \%$ Tween 80 ] in a dialysis bag (molecular cut-off, $10 \mathrm{kDa}$ ) at $37^{\circ} \mathrm{C}$ and under gentle magnetic stirring $(10 \mathrm{x} \mathrm{g})$. At fixed time intervals, $1 \mathrm{ml}$ supernatant was withdrawn and replaced with fresh buffer solution to maintain a sink condition. Furthermore, the amount of ZA released in the samples collected was extracted by acetonitrile and determined by HPLC using the aforementioned protocol. All experiments were performed in triplicate.

Cell culture. HFOb 1.19 cells were obtained from the American Type Culture Collection (Manassas, VA, USA) and cells were cultured for $24 \mathrm{~h}$ in Dulbecco's Modified Eagle's medium 
(DMEM) supplemented with $10 \%$ fetal bovine serum (Stem Cell Technologies, Seoul, Korea), $2 \mathrm{mM} \mathrm{L-glutamine,} 0.3 \mathrm{mg} / 1$ $\mathrm{G} 418,100 \mathrm{U} / \mathrm{ml}$ penicillin and $100 \mathrm{mg} / \mathrm{ml}$ streptomycin. Cells were maintained at $37^{\circ} \mathrm{C}$ with $5 \% \mathrm{CO}_{2}$ in a humidified incubator.

MTT assay. The cytotoxic potential of the individual formulations was evaluated using an MTT assay. Briefly, cells were seeded into 96 -well plates at a density of $0.5 \times 10^{4}$ cells/well in $0.1 \mathrm{ml}$ growth medium (Stem Cell Technologies) and incubated for $72 \mathrm{~h}$ at $37^{\circ} \mathrm{C}$. The following day, the cells were treated with the respective formulations [free ZA, ZA-loaded lipid bilayer (ZL) and HZL NPs] at concentrations between 0.001 to $50 \mu \mathrm{M}$ and also treated with blank formulation, where the blank liposome concentration was equivalent to the drug concentration of the formulation. Respective formulations were incubated for $72 \mathrm{~h}$ at $37^{\circ} \mathrm{C}$. At each time interval, the cells were washed twice with PBS and treated with MTT solution $(5 \mathrm{mg} / \mathrm{ml})$ and incubated for another $4 \mathrm{~h}$ at $37^{\circ} \mathrm{C}$. The resultant formazan crystals were then dissolved in $100 \mu$ l dimethyl sulfoxide (DMSO). The mixture was gently agitated in a microplate reader prior to measuring the absorbance at $570 \mathrm{~nm}$ and each experiment was repeated 6 times.

Apoptosis assay. Cell apoptosis was evaluated using the Annexin V/fluorescein isothiocyanate (FITC) apoptosis detection kit (BD Biosciences, Franklin Lakes, NJ, USA) and propidium iodide (PI; Sigma-Aldrich; Merck KGaA). Briefly, cells were harvested using $0.25 \%$ trypsin $48 \mathrm{~h}$ following treatment with different formulations, washed twice in cold PBS and re-suspended in binding buffer. Subsequently, cells were incubated with $5 \mu \mathrm{l}$ Annexin V/FITC and $5 \mu \mathrm{l}$ PI for $15 \mathrm{~min}$ at room temperature in the dark. A flow cytometer was used to measure the apoptosis of osteoblasts.

HA affinity test. In total, $3 \mathrm{mg}$ HZL NPs were dissolved in DMSO. Subsequently, $1 \mathrm{mg}$ nanocarrier in DMSO was added to $15 \mu \mathrm{l}$ trimethylamine. This solution was then mixed with $7.5 \mathrm{ml}$ distilled water, sonicated for $5 \mathrm{~min}$ at $37^{\circ} \mathrm{C}$ and dialyzed for $48 \mathrm{~h}$. A total of $2 \mathrm{ml}$ dialysis solution was stirred with $500 \mu \mathrm{l}$ HA suspension $(10 \mathrm{mg} / \mathrm{ml})$ in buffer solution. Following incubation, the suspension was centrifuged for $4 \mathrm{~min}$ at $2,571 \mathrm{x} \mathrm{g}$ and washed with buffer solution at room temperature. The supernatant was then collected and lyophilized and analyzed using a UV spectrophotometer at $280 \mathrm{~nm}$. HZL NPs without HA coating were used as a control.

Statistical analysis. All results are expressed as the mean \pm standard deviation. Data were analyzed using analysis of variance or the Student's t-test using IBM SPSS Statistics for Windows (version 14.0; SPSS Inc., Chicago, IL, USA) to determine whether differences between test groups were statistically significant. $\mathrm{P}<0.05$ was considered to indicate a statistically significant difference.

\section{Results}

Characterization of HZL NPs. Briefly, the schematic illustration depicted in Fig. 1 demonstrates the successful loading of hydrophilic ZA onto the aqueous core of the lipid nanoparticle

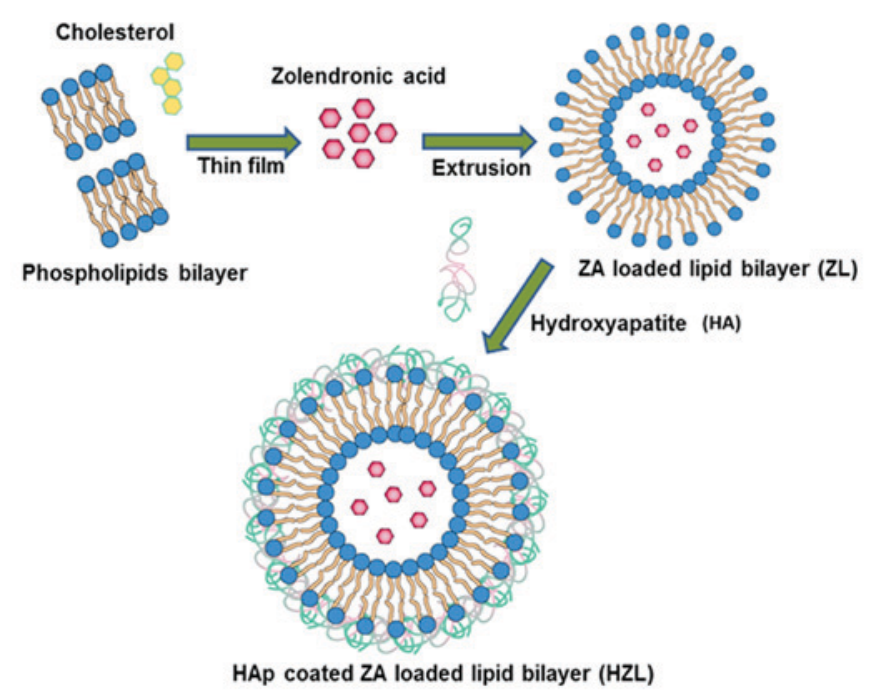

Figure 1. Schematic presentation of the preparation of hydroxyapatite coated zoledronic acid loaded lipid layer nanoparticles.

using the extrusion method. EPC and DOTAP were selected to prepare stable positively charged liposomes using the modified thin film method. An increase in the ratio of DOTAP in liposomes also leads to a more positive z-potential, since DOTAP itself is positively charged. Dynamic light scattering analysis is depicted in Fig. 2. The Z-potential of the liposome (3:1) was $12.4 \mathrm{mV}$ and the hydrodynamic size of the ZA loaded liposome was $172.4 \pm 3.47 \mathrm{~nm}$ (Fig. 2A). HA was fabricated on the surface of the lipid nanoparticle using the precipitation method and following HA precipitation, the mean particle size increased from 172.4 to $197.8 \mathrm{~nm}$ (Fig. 2C). Furthermore, following HA coating, the surface charge on the liposomes decreased from 12.4 to $-6.3 \mathrm{mV}$ (Fig. 2B and D). These results indicate that HA coating screens the negative charge of the liposomes. In addition, the loading efficiency of ZA was high ( $83 \%)$ corresponding to $9.28 \%$ drug loading due to the negative charge of the ZA interacting with the positively charged bilayer. This indicates a major contribution to the high encapsulation efficiency of ZA in ZL.

Surface morphology. The nanoparticle surface morphology of HZL was investigated using scanning electron microscopy (SEM). As observed in Fig. 3, HZL NPs were spherically shaped and uniformly distributed on the SEM grid. The SEM images of HZL revealed well-defined, spherical-shaped particles with a uniform distribution. The spherical nature of the particle was attributed to the self-assembly of the lipid bilayer. This figure reveals the SEM micrographs of particles of the HZL complex containing particles of various sizes (from a few microns to a few hundred microns). However, the overall size of the particles was larger as confirmed by scale bar and consisted of a smooth surface following coating with lipid as seen in SEM image.

In vitro drug release. As presented in Fig. 4, the amount of the drug cumulatively released from HZL was higher at a lower $\mathrm{pH}$ (5.0) than at physiological $\mathrm{pH}$ (7.4). The difference between the release of the drug at pH 5.0 and 7.4 was statistically significant at 10 to $50 \mathrm{~h}(\mathrm{P}<0.05$ and $\mathrm{P}<0.01)$. ZA-loaded HZL NPs 
A

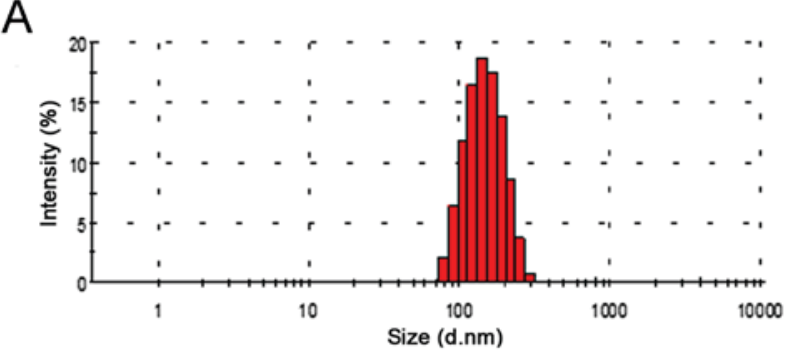

C

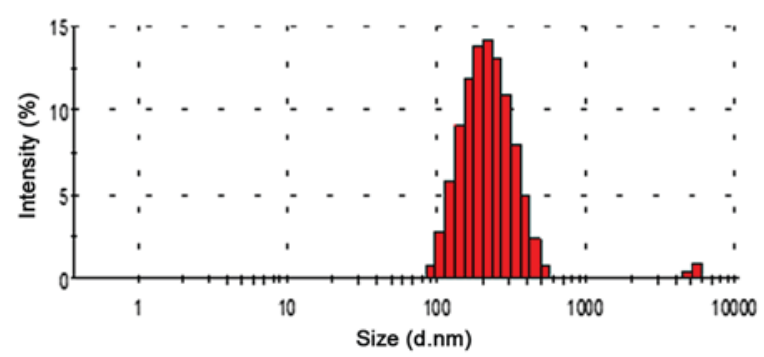

B

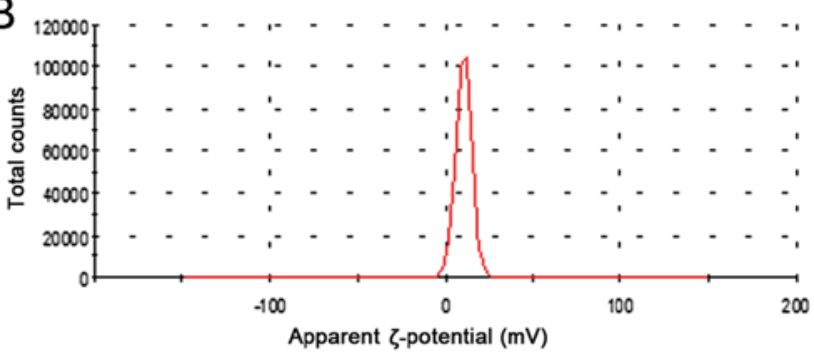

D

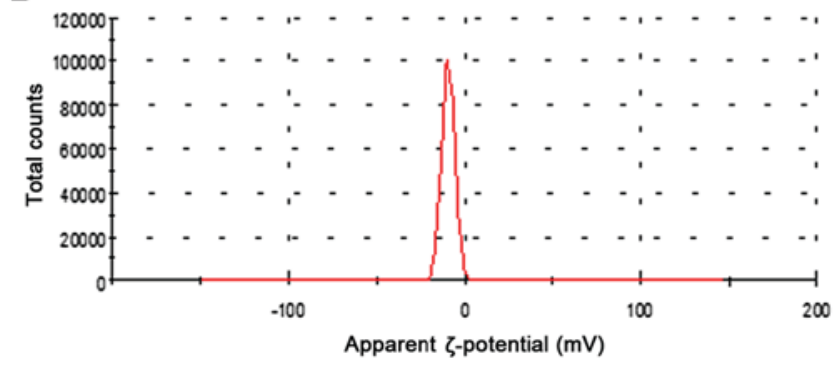

Figure 2. Dynamic light scattering analysis. (A) ZL particle size, (B) ZL $\zeta$-potential, (C) HZL particle size and (D) HZL $\zeta$-potential. HZL, hydroxyapatite-coated zoledronic acid loaded lipid bilayer; ZL, zoledronic acid loaded lipid bilayer.

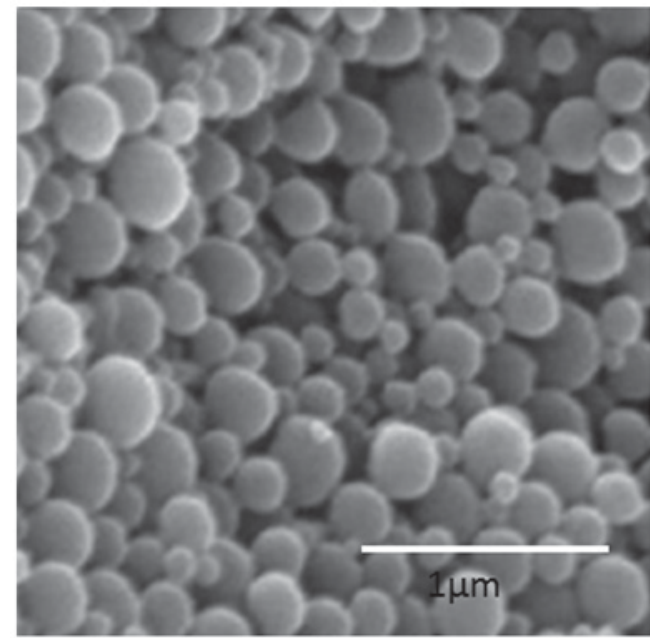

Figure 3. Scanning electron microscopy image of the hydroxyapatite-coated zoledronic acid loaded lipid bilayer nanoparticle (Scale bar, $1 \mu \mathrm{m}$ ).

at physiological $\mathrm{pH}$ exhibited a biphasic drug release pattern characterized by an initial rapid release in which $8.42 \pm 2.89 \%$ ZA was released over $6 \mathrm{~h}$, followed by a sustained continuous release phase in which $38.17 \pm 2.12 \% \mathrm{ZA}$ was released from the lipid bilayer nanoparticle over $48 \mathrm{~h}$. The $\mathrm{pH}$ around the border of osteoblasts is $~ 5.0$ during bone remodeling and homeostatic body fluid is 7.4 . HA more readily dissolves in acidic $\mathrm{pH}$ and under acidic conditions, there was an immediate burst release of $34.1 \pm 4.81 \%$ drug over $8 \mathrm{~h}$, due to the HA surface layer dissolving immediately. Consequently, drug release followed a sustained release profile from the nanoparticles, with $64.2 \pm 3.75 \%$ of drug being released following $48 \mathrm{~h}$.

In vitro cytotoxicity. The cytotoxicity of $\mathrm{ZA}, \mathrm{ZL}$ and $\mathrm{HZL}$ NPs was measured by an MTT assay. The viability of HFOb 1.19 cells remained $>80 \%$ when they were treated with different concentrations of a blank liposome (Fig. 5A). Furthermore,

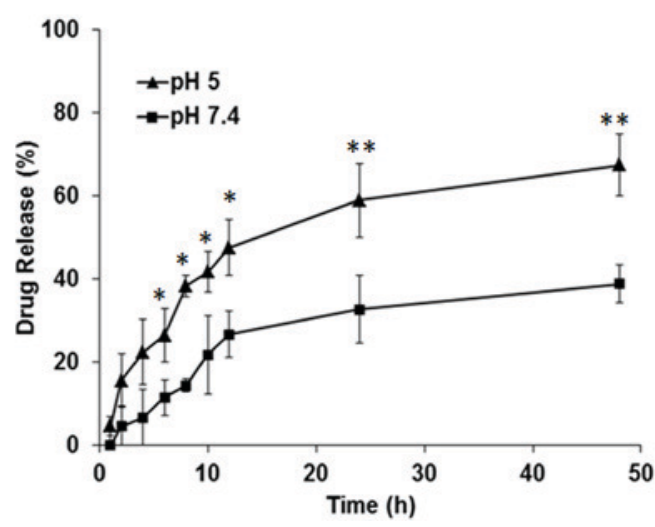

Figure 4. In vitro drug release study of zoledronic acid from hydroxyapatite coated zoledronic acid loaded lipid layer nanoparticles in PBS (pH 7.4) and acetic medium ( $\mathrm{pH} 5.0)$. ${ }^{*} \mathrm{P}<0.05$ and ${ }^{* *} \mathrm{P}<0.01$ vs. $\mathrm{pH} 7.4$.

the cytotoxicity of osteoblasts treated with HZL NPs was higher compared with cells treated with ZL-viability was $48 \%$ following treatment with $1 \mu \mathrm{M}$ HZL NP and incubation for 72 h (Fig. 5B). Furthermore, toxicity was dose-dependent, with higher concentrations of ZA, ZL and HZL NP inducing higher toxicity. Notably, HZL NP treatment resulted in significantly decreased viability when compared to that of free ZA at 0.1 to $50 \mu \mathrm{M}(\mathrm{P}<0.05)$. As expected, the osteoclast viability and differentiation were greatly affected by the presence of HZL NP and exhibited significantly higher cytotoxicity compared with the cytotoxicity induced by ZA and ZL following $48 \mathrm{~h}$ incubation. HZL NP may have induced higher toxicity in osteoblasts due to its higher cellular release of ZA.

Apoptosis assay. Annexin V/PI flow cytometric analyses were performed to determine whether the inhibition of cell viability differed following treatment with $\mathrm{ZA}, \mathrm{ZL}$ or $\mathrm{HZL}$ NP and to distinguish among healthy, early apoptotic and late apoptotic or necrotic cells in each group of cells. Qualitative 


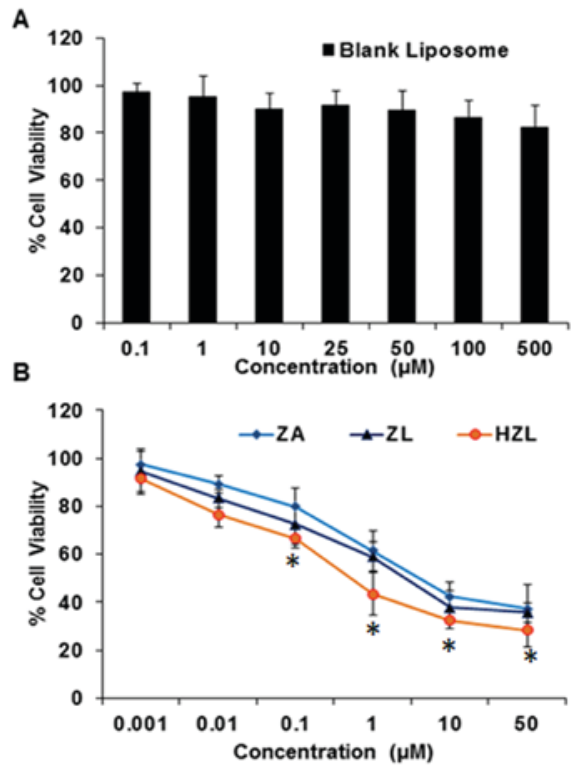

Figure 5. Cytotoxicity assay of (A) blank nanoparticles and (B) ZA, ZL and HZL NPs. ZL, ZA-loaded lipid bilayer; ZA, zoledronic acid; HZL NPs, hydroxyapatite-coated zoledronic acid loaded lipid bilayer nanoparticles. ${ }^{*} \mathrm{P}<0.05$ vs. ZA.

A
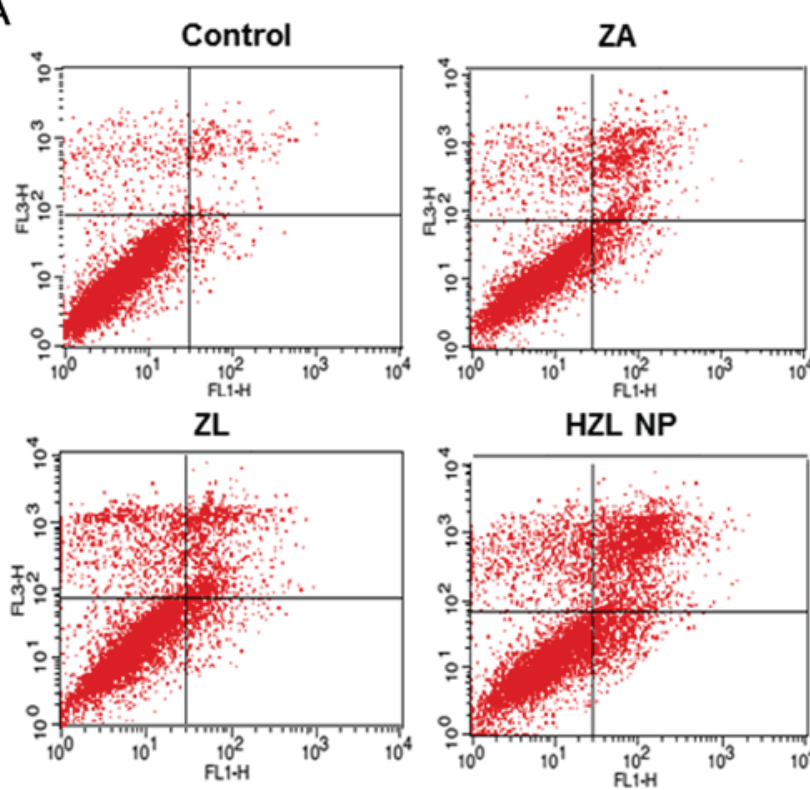

HZL NP

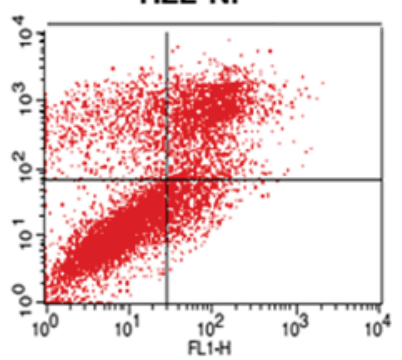

B

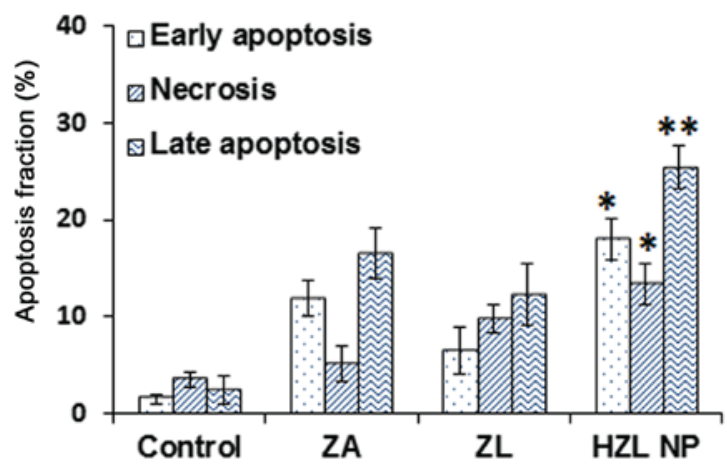

Figure 6. (A) Qualitative and (B) quantitative apoptosis analysis of ZA, ZL and HZL NPs by Annexin V/propidium iodide staining. ${ }^{*} \mathrm{P}<0.05$ and ${ }^{* *} \mathrm{P}<0.01$ vs. ZA. ZL, ZA-loaded lipid bilayer; ZA, zoledronic acid; HZL NPs, hydroxyapatite-coated zoledronic acid loaded lipid bilayer nanoparticles.

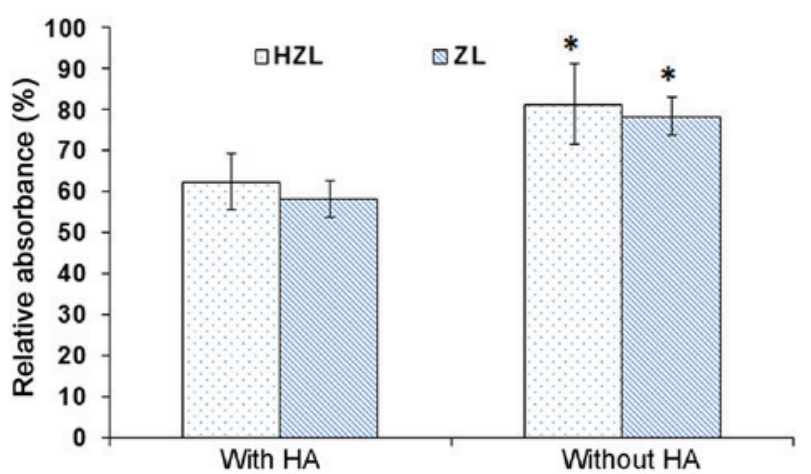

Figure 7. HA affinity assay on ZL and HZL NPs. "P<0.05 vs. with HA. ZL, Zoledronic acid loaded lipid bilayer; HZL NPs, hydroxyapatite-coated zoledronic acid loaded lipid bilayer nanoparticles; HA, hydroxyapatite.

and quantitative results demonstrated that following treatment with $10 \mu \mathrm{M} \mathrm{ZA}$ for 2 days, the proportion of apoptotic $\mathrm{HFOb}$ 1.19 cells in all treatment groups with ZA, ZL and HZL NP was significantly higher than that of the control group (Fig. 6). In cells cultured with ZA or ZL for 2 days, the percentage of viable cells decreased and the percentage of early apoptotic cells and late apoptotic or necrotic cells increased. However, cells treated with HZL NPs exhibited higher toxicity compared with cells treated with ZL; the proportion of early apoptotic cells increased from 7.5 \pm 2.1 to $18.1 \pm 12.4 \%(\mathrm{P}<0.05)$. Furthermore, the proportion of late apoptotic and necrotic cells increased significantly to $28.7 \pm 3.7$ and $16.1 \pm 1.2 \%$, respectively $(\mathrm{P}<0.01$ and $\mathrm{P}<0.05$, respectively) compared with cells treated with free ZA. These data were consistent with the trends of cell viability and indicate that the inhibition of cell viability by ZA released by HZL NP is due to the induction of apoptosis.

HA affinity test. To confirm the affinity of the nanoparticle to HA, a quantitative affinity test was performed (Fig. 7). HZL NPs were incubated with and without HA in buffer maintained at $\mathrm{pH}$ 7.4. The absorbance of HZL NP incubated with HA was $62 \%$. However, the absorbance of HZL NPs without HA was $82 \%$, resulting in a high amount of nanocarrier adhering to the surface of HA, due to the presence of HA on the surface of the NPs. The absorbance of ZL NPs was $\sim 57$ and $76 \%$ with and without HA, respectively. The absorbance of NPs was significantly higher in NPs incubated without HA compared with those incubated with HA (P<0.05; Fig. 7). ZA exhibited strong affinity with HA and the interactions between bisphosphates and HA involved the P-C-P backbone and also the hydroxyl groups (24). Notably, ZL exhibited affinity in the presence of HA as the ZA interacts with the HA conjugate on the surface, which may release and help to induce affinity towards HA whereas in the HZL NPs, the HA along with the drug may serve a vital role in stimulating adhesion. HZL NPs bind and accumulate in bone sites where the pores in the bone are 70-100 $\mathrm{nm}$ (25). Therefore, the NPs prepared with pores $\leq 100 \mathrm{~nm}$ can easily target and deliver drugs to the bone.

\section{Discussion}

OP is a progressive bone disease that makes the bone more porous (26). Bisphosphonates inhibiting osteoclast resorption 
are widely used in the prevention and treatment of the disease. Risedronate is a nitrogen-containing bisphosphonate approved by the Food and Drug Administration as a treatment of postmenopausal OP. Its primary mechanism is the inhibition of farnesyl diphosphate synthase, which ultimately results in the inhibition of osteoclast adhesion via osteoclast apoptosis on the margin of bone where absorption takes place (27). Lü et al (23) described the synthesis of the HA-nanofibre composite used to induce osteogenesis by the employment of mesenchymal stem cells (MSCs). The ability of HA to induce MSCs to differentiate into osteoblasts may be applicable in bone tissue engineering.

The present study therefore investigated the ability of HA-coated ZA-loaded lipid nanoparticle to inhibit the progression of OP. It has been hypothesized that HA coated nanoparticles may effectively inhibit osteoclasts and thereby improve the symptoms of OP. In the present study, liposomal systems were used, due to their inherent biocompatibility $(28,29)$. Liposomal systems were coated with HA by loading zoledronate in the lipid bilayer, the association between zoledronate and $\mathrm{HA}$ and the response of bone cells to these novel biomaterials was determined. The Z-potential of the DOTAP liposome (3:1) was $12.4 \mathrm{mV}$ and the hydrodynamic size of ZA-loaded liposome was $172.4 \pm 3.47 \mathrm{~nm}$. HA was then fabricated on the surface of the lipid nanoparticle using the precipitation method. As the HA was negatively charged, it electrostatically interacted with the positively charged lipid layer and formed a coat around the HZL NP. Additionally, the precipitation of $\mathrm{HA}$ on the surface of liposomes resulted in the formation of a core-shell structure. The negative charge of the liposomes was screened following HA coating. SEM images of HZL revealed well-defined, spherical-shaped particles with a uniform distribution.

ZA-loaded HZL NPs exhibited a biphasic drug release pattern characterized by an initial rapid release followed by a sustained continuous release from the lipid bilayer nanoparticle after $48 \mathrm{~h}$. HZL has numerous potential advantages as a bone drug delivery system due to its osteoconductivity. A switch from physiological $\mathrm{pH}$ to a lower $\mathrm{pH}$ around the inflamed sites may trigger drug release in these specific areas. Such sustained and prolonged release behavior may enhance the efficiency of OP therapy, as continually sustained drug release effectively prevents bone deformation during long-term treatment. Furthermore, it has been demonstrated that HZL NPs are optimized and the controlled release of ZA makes it suitable to treat OP. Blank nanocarriers exhibit lower cytotoxicity, which proves their biocompatibility and the nontoxic nature of the carrier used to deliver the drug to the diseased site. ZA-loaded HZL NPs increased the toxicity on osteoblasts indicating the potent antiresorptive action of ZA. The apoptosis rate in cells treated with HZL NPs is increased. ZA inhibits farnesyl diphosphate synthase, a key enzyme in the mevalonic acid pathway, and thereby inhibits the prenylation of small GTPases. This enzyme maintains the osteoclast function, and is widely used to treat bone disorders, including osteoporosis and metastatic bone disease. Small GTPases accumulate in the cells, which erroneously stimulate the downstream pathway, inhibiting osteoclast formation and inducing apoptosis (30). Thus, bone resorption mediated by osteoclasts is reduced, lowering the bone turnover rate and leading to a loss of bone mass.
In conclusion, in the current study HZL NPs were successfully prepared using a thin film and the extrusion method. The results demonstrated that the precipitation of HA on the surface was highly effective at treating the symptoms of OP. Furthermore, the nanosized hydrodynamic size and SEM image of the nanoparticle demonstrate that they are suitable for the delivery of ZA to specific sites in the bone. Additionally, a typical biphasic release pattern was observed with a sustained prolonged drug release exhibited throughout. Furthermore, higher concentrations of ZA induced cytotoxicity and exhibited a significant proportion of osteoclasts in the early and late phases of apoptosis. Therefore, HZL NPs exhibited a high selectivity for bone tissues and revealed a strong affinity towards HA, indicating that they may enhance therapeutic efficacy and may be developed as a novel method of treating patients with OP.

\section{Acknowledgements}

Not applicable.

Funding

No funding was received.

\section{Availability of data and materials}

The datasets used and/or analyzed during the current study are available from the corresponding author on reasonable request.

\section{Authors' contributions}

TG and SF were responsible for the formulation and initial characterization of nanoparticles. PS performed all the biological studies. YC designed the present study and wrote the manuscript.

\section{Ethics approval and consent to participate}

Not applicable.

\section{Patient consent for publication}

Not applicable.

\section{Competing interests}

The authors declare that they have no competing interests.

\section{References}

1. Akesson K: New approaches to pharmacological treatment of osteoporosis. Bull World Health Organ 81: 657-664, 2003.

2. Dhanwal DK, Dennison EM, Harvey NC and Cooper C: Epidemiology of hip fracture: Worldwide geographic variation. Ind J Orthop 45: 15-22, 2011.

3. Drake MT, Clarke BL and Lewiecki EM: The pathophysiology and treatment of osteoporosis. Clin Ther 37: 1837-1850, 2015.

4. Dalle Carbonare L, Zanatta M, Gasparetto A and Valenti MT: Safety and tolerability of zoledronic acid and other bisphosphonates in osteoporosis management. Drug Healthc Patient Saf 2: 121-137, 2010.

5. Rogers MJ, Crockett JC, Coxon FP and Mönkkönen J: Biochemical and molecular mechanisms of action of bisphosphonates. Bone 49: 34-41, 2011. 
6. Dunford JE, Thompson K, Coxon FP, Luckman SP, Hahn FM, Poulter CD, Ebetino FH and Rogers MJ: Structure-activity relationships for inhibition of farnesyl diphosphate synthase in vitro and inhibition of bone resorption in vivo by nitrogen-containing bisphosphonates. J Pharmacol Exp Ther 296: 235-242, 2001.

7. Salzano G, Marra M, Porru M, Zappavigna S, Abbruzzese A, La Rotonda MI, Leonetti C, Caraglia M and De Rosa G: Self-assembly nanoparticles for the delivery of bisphosphonates into tumors. Int J Pharm 403: 292-297, 2011.

8. Bellido T and Plotkin LI: Novel actions of bisphosphonates in bone: Preservation of osteoblast and osteocyte viability. Bone 49: 50-55, 2011.

9. Wei D, Jung J, Yang H, Stout DA and Yang L: Nanotechnology treatment options for osteoporosis and its corresponding consequences. Curr Osteoporos Rep 14: 239-247, 2016.

10. Barry M, Pearce H, Cross L, Tatullo M and Gaharwar AK: Advances in nanotechnology for the treatment of osteoporosis. Curr Osteoporos Rep 14: 87-94, 2016.

11. Ramasamy T, Ruttala HB, Gupta B, Poudal BK, Choi HG, Yong CS and Kim JO: Smart chemistry-based nanosized drug delivery systems for systemic applications: A comprehensive review. J Control Release 258: 226-253, 2017.

12. Peng H, Liu X, Wang G, Li M, Bratlie KM, Cochran E and Wang Q: Polymeric multifunctional nanomaterials for theranostics. J Mater Chem B 3: 6856-6870, 2015.

13. Ikoba U, Peng H, Li H, Miller C, Yu C and Wang Q: Nanocarriers in therapy of infectious and inflammatory diseases. Nanoscale 7 : 4291-4305, 2015.

14. Ruttala HB, Ramasamy T, Poudal BK, Choi Y, Choi JY, Kim J, Kwang Ku S, Choi HG, Soon Yong C and Oh Kim J: Molecularly targeted co-delivery of a histone deacetylase inhibitor and paclitaxel by lipid-protein hybrid nanoparticles for synergistic combinational chemotherapy. Oncotarget 8 : 14925-14940, 2017

15. Ruttala HB, Ramasamy T, Gupta B, Choi HG, Yong CS and Kim JO: Multiple polysaccharide-drug complex-loaded liposomes: A unique strategy in drug loading and cancer targeting. Carbohydr Polym 173: 57-66, 2017.

16. Wang Q, Cheng H, Peng H, Zhou H, Li PY and Langer R: Non-genetic engineering of cells for drug delivery and cell-based therapy. Adv Drug Deliv Rev 91: 125-140, 2015.

17. Meng S, Su B, Li W, Ding Y, Tang L, Zhou W, Song Y and Caicun Z: Integrin-targeted paclitaxel nanoliposomes for tumor therapy. Med Oncol 28: 1180-1187, 2011.

18. Ruttala HB, Ramasamy T, Shin BS, Choi HG, Yong CS and Kim JO: Layer-by-layer assembly of hierarchical nanoarchitectures to enhance the systemic performance of nanoparticle albumin-bound paclitaxel. Int J Pharm 519: 11-21, 2017.

19. Xu Q, Tanaka Y and Czernuszka JT: Encapsulation and release of a hydrophobic drug from hydroxyapatite coated liposomes. Biomaterials 28: 2687-2694, 2007.
20. Venkatesan P, Puvvada N, Dash R, Prashanth Kumar BN, Sarkar D, Azab B, Pathak A, Kundu SC, Fisher PB and Mandal M: The potential of celecoxib-loaded hydroxyapatite-chitosan nanocomposite for the treatment of colon cancer. Biomaterials 32 3794-3806, 2011.

21. Iafisco M, Ruffini A, Adamiano A, Sprio S and Tampieri A: Biomimetic magnesium-carbonate-apatite nanocrystals endowed with strontium ions as anti-osteoporotic trigger. Mater Sci Eng C Mater Biol Appl 35: 212-219, 2014.

22. Pilia M, Guda T and Appleford M: Development of composite scaffolds for load-bearing segmental bone defects. BioMed Res Int 2013: 458253, 2013.

23. Lü LX, Zhang XF, Wang YY, Ortiz L, Mao X, Jiang ZL, Xiao ZD and Huang NP: Effects of hydroxyapatite-containing composite nanofibers on osteogenesis of mesenchymal stem cells in vitro and bone regeneration in vivo. ACS Appl Mater Interfaces 5: 319-330, 2013.

24. Jueng H, Sung KC, Eun SP, Kun H, Hee DH and Byung CS: Enhanced stability of hydroxyapatite-coated liposomes for ultrasound-triggered drug release. Bull Korean Chem Soc 36: 83-87, 2015

25. Yewle JN, Puleo DA and Bachas LG: Enhanced affinity bifunctional bisphosphonates for targeted delivery of therapeutic agents to bone. Bioconjug Chem 22: 2496-2506, 2011.

26. Wang D, Miller S, Sima M,Kopecková P and Kopecek J: Synthesis and evaluation of water-soluble polymeric bone-targeted drug delivery systems. Bioconjug Chem 14: 853-859, 2003.

27. Cummings SR and Melton LJ: Epidemiology and outcomes of osteoporotic fractures. Lancet 359: 1761-1767, 2002.

28. Nishikawa M, Akatsu T, Katayama Y, Yasutomo Y, Kado S, Kugal N, Yamamoto M and Nagata N: Bisphosphonates act on osteoblastic cells and inhibit osteoclast formation in mouse marrow cultures. Bone 18: 9-14, 1996

29. Gaspar MM, Gobbo O and Ehrhardt C: Generation of liposome aerosols with the Aeroneb Pro and the AeroProbe nebulizers. J Liposome Res 20: 55-61, 2010.

30. Dunford JE, Rogers MJ, Ebetino FH, Phipps RJ and Coxon FP: Inhibition of protein prenylation by bisphosphonates causes sustained activation of Rac, $\mathrm{Cdc} 42$, and Rho GTPases. J Bone Miner Res 21: 684-694, 2006.

This work is licensed under a Creative Commons Attribution-NonCommercial-NoDerivatives 4.0 International (CC BY-NC-ND 4.0) License. 